\title{
Modified Different Neighbor History Spray and Wait using PROPHET in Delay Tolerant Network
}

\author{
Anjula Mehto \\ M.Tech Scholar \\ Department of C.S.E. \\ MANIT Bhopal, 462015 India
}

\author{
Meenu Chawla, Ph.D \\ Professor \\ Department of C.S.E. \\ MANIT Bhopal, 462051 India
}

\begin{abstract}
Delay Tolerant Networks or Disruption Tolerant Networks are intermittent networks that may lack continuous connectivity as most of the time there does not exist a direct link between source and destination for message transmission. In this paper, Modified DNH (Different Neighbor-History) Spray and Wait using PROPHET is proposed. It improves the wait phase of Different Neighbor-History Spray and Wait. This protocol calculates number of message copies to be forwarded based on performance of receiver node in spray phase and in wait phase the waiting node uses probabilistic routing protocol using history of encounters and transitivity for transmission. Simulation results show that this method improves delivery probability and average latency of transmission as compared to Spray and Wait and also DNH-Spray and Wait routing scheme.
\end{abstract}

\section{General Terms}

Delay Tolerant Network, Opportunistic Network Environment Simulator

\section{Keywords}

Routing protocols, Spray and wait routing, PROPHET routing

\section{INTRODUCTION}

Delay Tolerant Network (DTN) [1] is an opportunistic network where message transmission depends on contact between mobile nodes and transfer opportunities are of limited duration. There are many real networks that follow DTN, for example, inter-planetary networks (IPN) [2], under water sensor networks [3], wildlife racking and habitat monitoring sensor networks [4], satellite networks [5], etc. Transmission delay can be very large and unpredictable in DTN because of intermittent and unstable connection between nodes. Connection between nodes may change or break soon after it has been discovered, even while being discovered.

To deal with this situation, DTN uses store and forward mechanism to send a message. The DTN architecture implements store and forward mechanism by a new protocol called bundle protocol [1].

The rest of paper is organized as follows. Section II presents review of some related work on DTN routing protocols. Section III describes in detail the current work on Modified DNH-Spray and Wait using PROPHET. Section IV describes the simulation setup. Section V shows the results to analyze the routing protocols and conclusion is given in section VI.

\section{RELATED WORK IN DTN ROUTING PROTOCOLS}

This section reviews some of the well known routing protocol in DTNs.

\subsection{Epidemic Routing}

Historically the first protocol in DTN is Epidemic routing [6]. In Epidemic routing every node continuously replicates messages to newly arrived nodes that do not already have the message copy.

\subsection{PROPHET Routing}

In PROPHET [7], message forwarding is based on probability calculation by every node to each destination node. This probability is called delivery predictability. When two nodes are encountered, messages are forwarded to a node that has higher delivery predictability to the destination. Delivery predictability $P_{(a, b)} \in[1,0]$ for each node A to each known destination $\mathrm{B}$. This delivery predictability shows how likely it is that this node will deliver a message to destination. When two nodes meet they also exchange their delivery predictability information for known destination with summary vector. The information in summary vector is used to decide which message should be request from other node and the delivery predictability information is used to update internal delivery predictability vector of receiver node. The message exchange decision is based on a forwarding strategy. That is, when two nodes meet, a message is transferred to other node, if the delivery predictability of the destination of message is higher at the other node [7].

Delivery predictability calculation has three parts.

I. Update the delivery predictability metric, whenever a node is encountered. This is necessary because nodes that are often encountered have high delivery predictability. The Eq.1 shows this calculation.

$$
\mathrm{P}_{(\mathrm{a}, \mathrm{b})}=\mathrm{P}_{(\mathrm{a}, \mathrm{b}) \text { old }}+\left(1-\mathrm{P}_{(\mathrm{a}, \mathrm{b}) \text { old }}\right) \times \mathrm{P}_{\text {init }}
$$

Where $P_{\text {init }} \in[1,0]$ is an initialization constant

II. The delivery predictability must age because if two nodes do not encounter each other in a while, then they are less likely to forward messages to each other. Eq.2 shows ageing equation.

$$
\mathbf{P}_{(\mathbf{a}, \mathbf{b})}=\mathbf{P}_{(\mathbf{a}, \mathbf{b}) \text { old }} \times \gamma^{\mathbf{k}}
$$

Where $\gamma^{k} \in[1,0)$ is an aging constant.

III. The delivery predictability also follow the transitive property, that is if a node A frequently encounters node $\mathrm{B}$ and node $\mathrm{B}$ frequently encounters node $\mathrm{C}$ then node $\mathrm{C}$ probably is a good node to forward message destined for node A. Eq. 3 shows effect of transitivity on delivery predictability.

$\mathrm{P}_{(\mathrm{a}, \mathrm{c})}=\mathrm{P}_{(\mathrm{a}, \mathrm{c}) \text { old }}+\left(1-\mathrm{P}_{(\mathrm{a}, \mathrm{c}) \text { old }}\right) \times \mathrm{P}_{(\mathrm{a}, \mathrm{b})} \times \mathrm{P}_{(\mathrm{b}, \mathrm{c})} \times \beta$

Where $\beta €[0,1]$ is a scaling constant that decides how large impact the transitivity should have on the delivery predictability. 


\subsection{Spray and Wait Routing}

Spray and Wait (SaW) [8] is an improvement of Epidemic routing which has controlled flooding. It controls the blind forwarding strategy of Epidemic routing by associating a number of message copies $\mathrm{L}$ to the generated message at source node. L shows the maximum allowable copies of a message in the network.

SaW has two phases: (1) Spray phase and (2) Wait phase. The source node initially has $\mathrm{L}$ number of copies of a message. In the spray phase the source node forwards L copies to $\mathrm{L}$ distinct nodes. If all these $\mathrm{L}$ nodes are not the destination then they enter into the wait phase till the direct transmission to the destination.

Binary Spray and Wait (BSW) [8] improves SaW. In Binary Spray and Wait the source of a message initially starts with $\mathrm{L}$ copies. When it encounters first node with no copies then it hands over $\lceil L / 2]$ copies to that node and $[L / 2\rfloor$ keeps. Now this process is repeated for both source and relay that has $\mathrm{L}>$ 1 message copies, and when the node either is left with only one copy, it switches to wait phase and wait till the direct transmission to the destination.

However Saw and binary Spray and Wait forward constant L number of copies i.e. blindly forward message copies to relay node without calculating performance factor of the node.

\subsection{Different Neighbor History (DNH) Spray and Wait Routing}

The Different Neighbor History Spray and Wait (DNH-SaW) Routing [9] improves SaW. Instead of forwarding constant number of L message copies, DNH-SaW dynamically calculates number of message copies $\mathrm{L}$ to be forwarded based on performance factor of encountered node in spray phase.

This protocol calculates performance factor based on finding a receiver node that has high performance and should receive more message than other receiver nodes. After the performance factor calculation the source node or relay node forward a number of message copies to a receiver node based on performance factor calculation.

\subsubsection{Spray phase}

The spray phase consists of two parts: (1) node performance calculation and (2) forwarding strategy

\subsubsection{Node performance calculation}

Objective of this part is to find node performance factor of receiver node that has high performance and should receive more message copies than other node. Node performance calculation is motivated by set cover problem described in [10]. The main work of this part is to find a complete set of neighbor history of encountered nodes. The complete set is the union of neighbor list of encountered nodes. This set tells about number of nodes that the two nodes cover in that area.

Figure 1 describes the method to find the complete set. Before node $\mathrm{P}$ encounters node $\mathrm{Q}$, node $\mathrm{P}$ has ever encountered node $\mathrm{A}$ and $\mathrm{B}$, while node $\mathrm{Q}$ has encountered node $\mathrm{D}, \mathrm{E}, \mathrm{J}$ and $\mathrm{X}$. After this when node $\mathrm{P}$ and $\mathrm{Q}$ encounters each other they update their neighbor history list and node $\mathrm{P}$ add $\mathrm{Q}$ and node $\mathrm{Q}$ adds node $\mathrm{P}$ into their lists. Now node $\mathrm{P}$ and $\mathrm{Q}$ calculate complete set that is A, B, D, E, J, P, Q, X.

The repeating behavior of real user node [7] supports this idea and when node $\mathrm{P}$ forwards messages to node $\mathrm{Q}$ then in future the messages are likely to cover all the nodes in the complete set.

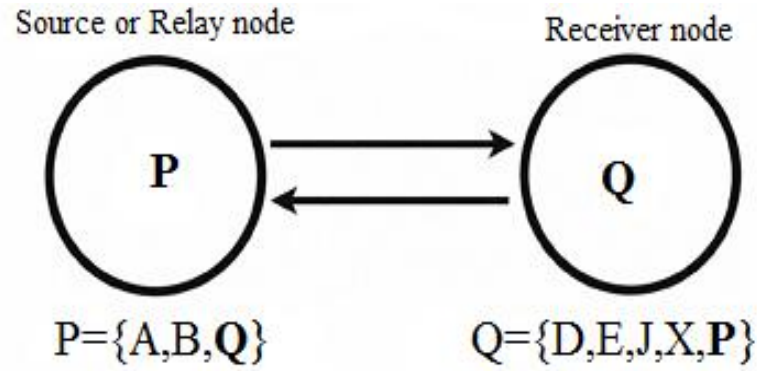

Fig 1: Exchange of neighbor list to calculate complete set

Node $\mathrm{P}$ forwards a number of message copies to node $\mathrm{Q}$ derived from the performance of encountered node $\mathrm{Q}$. The performance of source node or relay node is the ratio of number of nodes in the receiver's node neighbor history that the source node or relay node never met to total number of member in complete set. The performance of a receiver node is:

$$
\mathrm{PF}=\mathrm{V} / \mathrm{S}
$$

Where $P F$ is the performance factor of a receiver node, $\mathrm{V}$ is the number of nodes in the Q's neighbor history that $P$ has never met and $\mathrm{S}$ is the total number of members in the complete set.

\subsubsection{Forwarding strategy:}

Source node or relay node with more than one message copy will repeatedly forwards the message copy to other receiver node by the forwarding strategy given in this section. This source or relay node forwards the message copy until each node left with only one message copy.

Source node keeps L copies of every generated message. When a source node or relay node encounters a receiver node then they update their neighbor history list and exchange this list with each other. The number of message copies calculated by source or relay node to be forwarded to encountered node $\mathrm{Q}$ is:

$$
N_{2}=\left\lceil P F \times N_{1}\right\rceil
$$

Where PF is the performance factor of receiver node, $N_{2}$ is the number of copies to be forwarded to receiver node $\mathrm{Q}$ and $N_{1}$ is the number of message copies that node $\mathrm{P}$ has before forwarding.

After forwarding, node $\mathrm{P}$ has remained number of copies:

$$
N_{1}^{\prime \prime}=N_{1}-N_{2}
$$

Where $N_{1}^{\prime \prime}$ is a number of copies that node P keeps, $N_{1}$ is a number of copies that node $\mathrm{P}$ has before forwarding and $N_{2}$ is the number of copies that should be forwarded to node $\mathrm{Q}$.

\subsubsection{Wait phase}

When a node either source or relay node is left with only one message copy then it switches into the wait phase. In this phase the node waits till the direct transmission to the destination.

This DNH-SaW protocol reduces overhead than previous and also improve delivery probability.

\subsection{Other Works}

Jingfeng et al. proposed Spray and Wait Routing Based on Average Delivery Probability in Delay Tolerant Network [11]. It avoids blind forwarding scheme of $\mathrm{SaW}$ routing. It provides 
average delivery predictability based message forwarding in wait phase. It improves delivery rates and minimum average latency than SaW and BSW.

Yong-Pyo et al. proposed Composite Methods for Improving Spray and Wait Routing Protocol in Delay Tolerant Networks [12].It uses ACK message and delivery predictability based message forwarding in wait phase. ACK message is used to manage limited buffer size at relay nodes to avoid the drop of important message. It provides delivery rate and lower overhead ratio compared to Epidemic, PROPHET and SaW.

Guizhu et al. proposed Dynamic Spray and Wait Routing algorithm with Quality of Node in Delay Tolerant Network [13]. It introduces the notion of QoN (Quality of Nodes) to forward the number of message copies dynamically to avoid blind forwarding scheme in BSW. QoN indicates that one node meets other different nodes within a given time interval. It improves the delivery ration and reduces the average delay and network overhead as compare to Epidemic and BSW routing protocols.

\section{MODIFIED DIFFERENT NEIGHBOR HISTORY SPRAY AND WAIT USING PROPHET}

The Modified Different Neighbor-History Spray and Wait using PROPHET enhances the wait phase of DNH-SaW based on the delivery predictability calculation of PROPHET. Instead of waiting for direct transmission to the destination, the protocol use delivery probability to forward message copy to a node having higher delivery probability.

The performance of DNH-SaW is better in terms of delivery ratio or rate and overhead ratio but the average latency is higher than BSW. This is because it uses direct delivery scheme in wait phase. Often the direct delivery to the destination creates a problem in successful transmission of message in DTN. This may happen that the direct transmission to the destination will not occur because of link failure or Time to Live (TTL) of massage may expire due to long delay in meeting to the destination.

The protocol having two phases:

(1) Spray phase forwards $\mathrm{N}$ number of message copy based on performance factor calculation.

(2) In the Wait phase a node having single message copy forwards message to node having higher delivery probability for transmission to destination.

\subsection{Spray Phase}

This phase is same as DNH-SaW's spray phase. The objective of this phase is to find out node performance. The node performance calculation motivated by set cover problem. The basic idea of this phase is to forward message to cover all nodes in a particular area by minimum number of transmission. This phase finds complete set neighbor history of the two nodes that are encountered. This phase also updates delivery predictability using PROPHET.

\subsection{Forwarding using PROPHET in Wait Phase}

When nodes either relay or source node are left with only one message copy, it switches to wait phase. In this scheme the node can forward the message to the other node with the higher delivery predictability to the destination. The usability of delivery probability can increase the possibility that enables intermediate node to relay the messages between two nodes that cannot contact at all.

\subsection{Algorithm}

a) Spray Phase:

Exchange Neighbor-history list to find complete set and delivery predictability

Calculate

1. Performance factor

$$
P F=V / S
$$

2. Calculate number of copies forwarded by node $\mathrm{A}$ to encounter node B.

$$
N_{2}=\left\lceil P F \times N_{1}\right\rceil
$$

3. Calculate number of copies which node A keeps

$$
N_{1}^{\prime \prime}=N_{1}-N_{2}
$$

IF the number of spray message $(\mathrm{N})$ is larger than 1

THEN replicate $\mathrm{N} 2$ number of message copy, modify $N=N_{1}^{\prime \prime}$

END IF

UPDATE Delivery probability using PROPHET

$P_{(a, b)}=P_{(a, b) \text { old }}+\left(1-P_{(a, b) \text { old }}\right) \times P_{\text {init }}$

$P_{(a, b)}=P_{(a, b) \text { old }} \times \gamma^{k}$

$P_{(a, c)}=P_{(a, c) o l d}+\left(1-P_{(a, c) o l d}\right) \times P_{(a, b)} \times P_{(b, c)} \times \beta$

b) Forwarding using PROPHET in wait phase:

IF for a node ' $\mathrm{P}$ ' delivery probability of $\mathrm{P}(\mathrm{b}, \mathrm{d})>\mathrm{P}(\mathrm{a}, \mathrm{d})$ THEN forward the message to the node $b$ ELSE store and carry the message till the next encounter END IF

\section{SIMULATION SETUP AND PERFORMANCE EVALUATION 4.1 Model}

Several assumptions are considered for simulation. Every node has an infinite buffer to generate message and a finite size buffer to store intermediate message as relay node. Contact duration or transmission opportunities are limited and there has been frequent link disconnect during transmission. A node does not have prior knowledge of network connectivity and no control over node movement. First in first out (FIFO) policy has been implemented for message dropping and scheduling in the node's buffer.

\subsection{Simulation Environment}

To test the performance of the proposed protocol, the protocol and other Compared protocols have been implemented in Opportunistic Network Environment (ONE) simulator [14]. The simulation environment uses $4500 \times 3400$ meters area of the Helsinki city. Two experiments have been conducted with different simulation parameters. Table 1 and table 2 show the simulation parameters for two different experiments to realize the performance of protocol. First set of experiment based on varying simulation time. Second set of experiment based on varying buffer size. 


\subsubsection{Simulation setup to analyze impact of varying simulation time on performance metric}

This simulation scenario is based on varying simulation time to realize the performance of Modified Different NeighborHistory Spray and Wait Integrated with PROPHET with other protocols. Three different types of mobile nodes have been used in this simulation scenario; pedestrians, cars, and trams. Node movement of pedestrians and cars is Shortest Path Map Based movement and a tram uses Routed Map Based movement. Simulation time is 10 hours, and the message TTL is 4 hours. A message generation interval is 25-35 seconds. The message size is varied from $100 \mathrm{kB}$ to $200 \mathrm{kB}$. The initial number of message copy (L copy) generated at source node is 10. All the other simulation parameters are shown in table 1.

\subsubsection{Simulation setup to analyze impact of}

varying buffer size on performance metric

This simulation scenario is based on varying buffer size to realize the performance of our protocol with other protocols. Two types of mobile nodes; pedestrians and cars have been used in this simulation scenario. The message TTL is 4 hours. A message generation interval is $25-35$ seconds. The initial number of message copy (L copy) generated at source node is 10. The message size is varied from $500 \mathrm{kB}$ to $1 \mathrm{MB}$. All the other simulation parameters are shown in table 2 .

Table 1.Simulation parameters

\begin{tabular}{|c|c|c|c|}
\hline \multirow{2}{*}{ Parameters } & \multicolumn{3}{|c|}{ Node Type } \\
\cline { 2 - 4 } & Pedestrians & Cars & Trams \\
\hline $\begin{array}{c}\text { Number of } \\
\text { nodes }\end{array}$ & 70 & 30 & 6 \\
\hline $\begin{array}{c}\text { Movement } \\
\text { model }\end{array}$ & $\begin{array}{c}\text { Shortest Path } \\
\text { Map-Based }\end{array}$ & $\begin{array}{c}\text { Shortest } \\
\text { Path Map- } \\
\text { Based }\end{array}$ & $\begin{array}{c}\text { Routed } \\
\text { Map- } \\
\text { Based }\end{array}$ \\
\hline Wait time (s) & $10-30$ & $10-30$ & $50-100$ \\
\hline Speed (m/s) & $0.5-1.5$ & $5.6-16.7$ & $10-22$ \\
\hline Buffer size & 10 & 10 & 50 \\
\hline $\begin{array}{c}\text { Transmission } \\
\text { range (m) }\end{array}$ & 10 & 10 & 50 \\
\hline
\end{tabular}

Table 2 Simulation parameters

\begin{tabular}{|c|c|c|}
\hline \multirow{2}{*}{ Parameters } & \multicolumn{2}{|c|}{ Node Types } \\
\cline { 2 - 3 } & Pedestrians & Cars \\
\hline Number of nodes & 100 & 100 \\
\hline Simulation time & \multicolumn{2}{|c|}{ 6 hours } \\
\hline Node movement & Shortest Path Map Based \\
\hline Node Speed (m/s) & $0.5-1.5$ & $6-12$ \\
\hline $\begin{array}{c}\text { Packet transmission } \\
\text { speed }\end{array}$ & \multicolumn{2}{|c|}{250 Kbyte/s } \\
\hline Transmission range & \multicolumn{2}{|c|}{$10 \mathrm{~m}$} \\
\hline Buffer Size & \multicolumn{2}{|c|}{$-5 \mathrm{M}$} \\
\hline
\end{tabular}

\section{SIMULATION RESULTS}

The results presented in this paper are average of 5 simulation runs for 5 different seed values.

In this paper the proposed method compared with BSW and DNH-SaW routing protocols. The comparison is based on three performance metrics: delivery ratio or delivery rate, overhead ratio and average latency.

\subsection{Performance Metrics}

The three metrics to measure the performance of the different protocols [15]:

\subsubsection{Delivery ratio}

The delivery ratio is the ratio of total number of messages delivered to their destination to total number of created messages at source node.

$$
\text { Delivery ratio }=\frac{D}{G}
$$

$D$ is a number of messages delivered to destination, and $G$ is a number of created messages.

\subsubsection{Overhead ratio}

The overhead ratio reflects how many redundant packets are relayed to deliver one packet. It simply reflects transmission cost in a network.

$$
\text { Overhead ratio }=\frac{R-D}{D}
$$

$R$ is a number of messages forwarded by relay nodes, and $D$ is a number of messages delivered to their destination.

\subsubsection{Average delay}

The average delay is the time between messages is created and the messages are received at a destination.

$$
\text { Average delay }=\frac{\sum_{\mathrm{i}=1}^{\mathrm{n}}\left(\mathrm{R}_{\mathrm{i}}-\mathrm{G}_{\mathrm{i}}\right)}{\mathrm{n}}
$$

$n$ is a number of messages delivered to their destinations, $R_{i}$ is the time when a message $i$ reaches to its destination, and $G_{i}$ is the time when a message $i$ is created.

\subsection{Results to Analyze Impact of Varying Simulation Time on Performance Metrics}

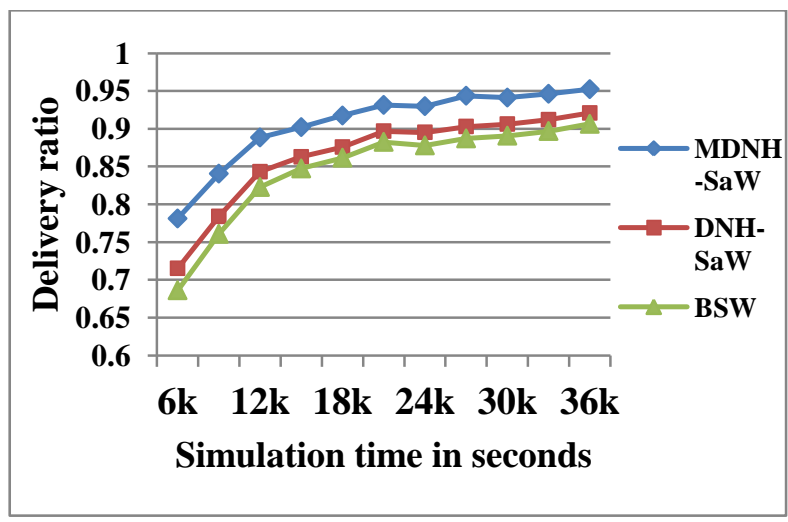

Fig 2: Impact of varying simulation time on delivery ratio

Figure 2 shows delivery ratio of Modified DNH-SaW using PROPHET compared to other protocols. For all protocols the delivery ratio increases in accordance with the increase in simulation time. This is because as simulation time increases nodes cover more area and deliver more messages to destination. Figure 2 shows improvement in delivery ratio of MDNH-SaW using PROPHET over other two protocols because of improved spray and wait phase. The node performance calculation in spray phase limits the blind forwarding message strategy of $\mathrm{SaW}$ and the usability of delivery predictability increase the possibility of successful transmission in wait phase.

Figure 3 shows average latency comparison of all the three protocols. For all protocols the average latency increases in accordance with the increase in simulation time. This is because of network congestion and consequently, a message takes more time to reach the destination. The latency of 
proposed scheme is better than other two protocols. This is because of modified wait phase of the propose scheme. In the wait phase the node with only one copy does not wait for direct transmission to destination and forwards message to a node having higher delivery predictability to destination. In this way the packets reached to the destination with better average latency than BSW and DNH-SaW.

The average latency of DNH-SaW is higher than BSW because it uses less number of network resources for message forwarding.

Figure 4 shows that the overhead of the proposed scheme is higher than BSW and DNH-SaW. This is because the proposed scheme uses probability forwarding scheme at wait phase and this increase the number of relays and hence increase in overhead ratio. Although the proposed scheme has shortcoming in terms of network cost or overhead ratio but delivery ratio of the scheme has improved and the delivery ratio is important metric that other in DTNs. The overhead ratio of $\mathrm{DNH}-\mathrm{SaW}$ is less than $\mathrm{BSW}$ because it uses less number of messages forwarding in spray phase than BSW.

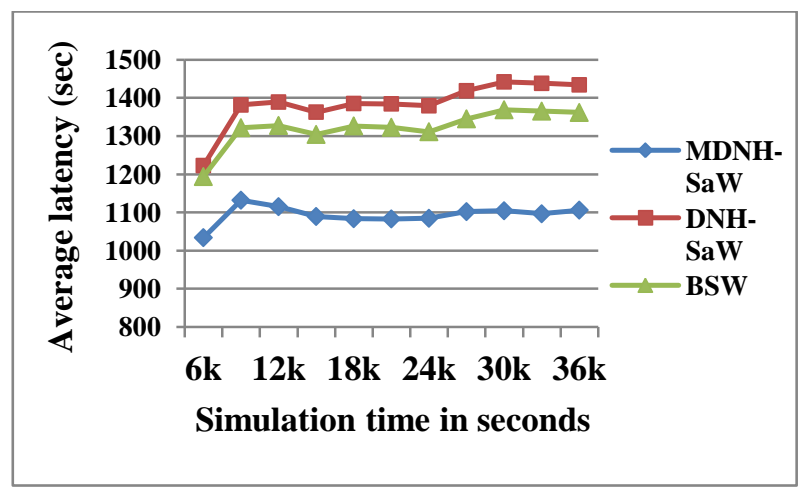

Fig3: Impact of varying simulation time on average latency

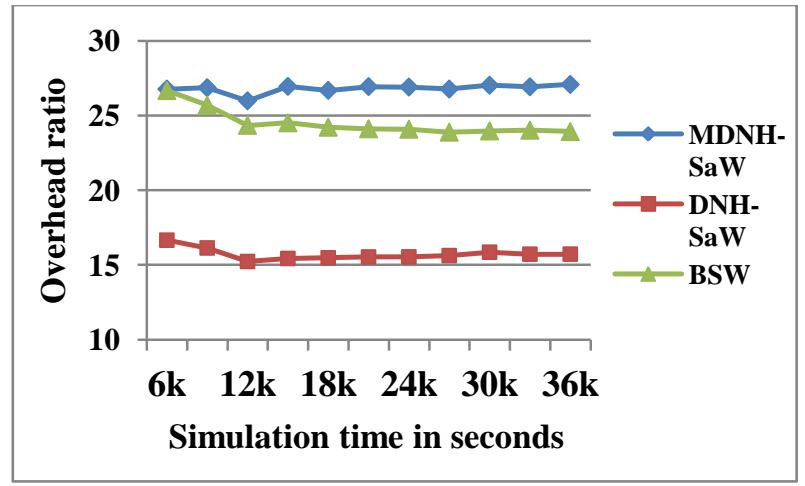

Fig 4: Impact of varying simulation time on overhead ratio

Figure 5 shows delivery ratio comparison of all the three protocols. Result shows that as buffer size increase all the three protocols show improvement in delivery ratio. This is because of reduction in message and hence more packets reached to their destination. The proposed scheme shows better delivery ratio than other two protocols because of modified wait phase. The figure shows that for small network after $2 \mathrm{M}$ buffer size the delivery ratio is constant for proposed scheme. This shows that for small networks this scheme is better with small buffer size.

Figure 6 shows comparison of average delay for all the three protocols. All the three protocols shows improvement in average delay as buffer size increase because reduction in message drop increase delivery of messages to destination. The proposed scheme shows better average delay as compared to BSW and DHN-SaW as buffer size increases. This is because usability of delivery predictability scheme at wait phase.

Figure 7 shows overhead comparison of BSW, DNH-SaW and modified DNH-SaW using PROPHET. Result shows that overhead of proposed scheme is higher than BSW and DNH$\mathrm{SaW}$ because number of relays increase in wait phase due to predictability based forwarding.

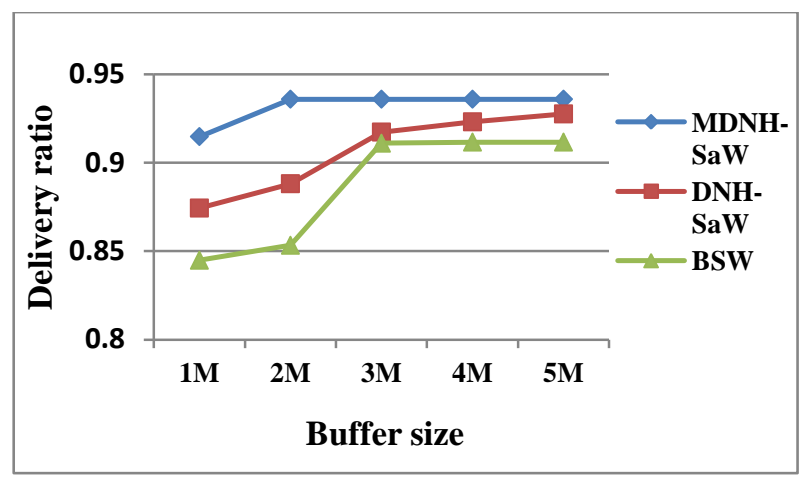

Fig 5: Impact of varying buffer size on delivery ratio

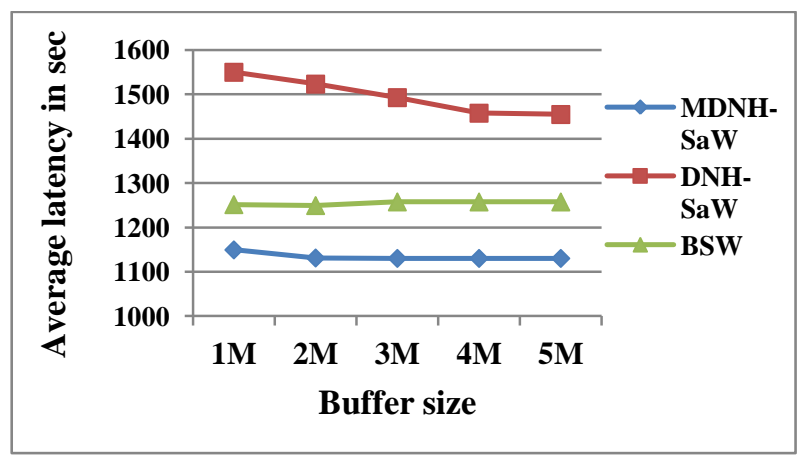

Fig 6: Impact of varying buffer size on average latency

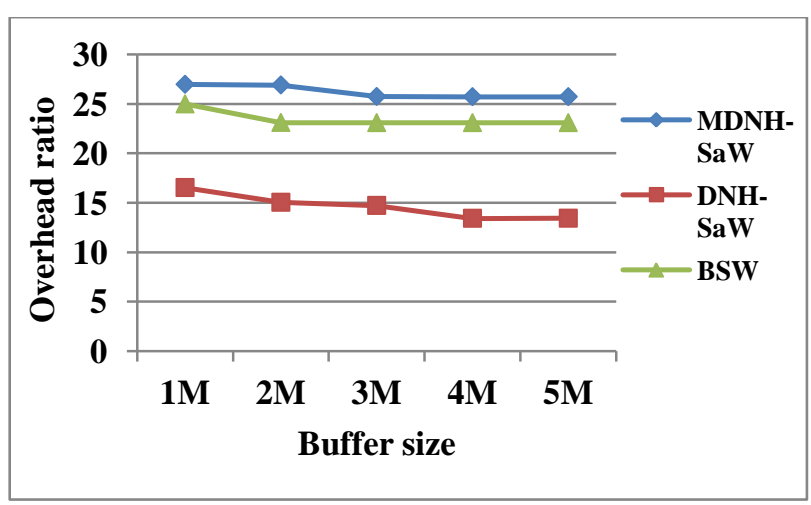

Fig 7: Impact of varying buffer size on overhead ratio

\section{CONCLUSION}

The DTN is a special type of mobile ad-hoc network. In DTN most of the message delivery is affected by frequent link disconnection and long delivery delay. The log delay sometimes measured in hours or days.

In this paper Modified DNH-SaW using PROPHET has been proposed. This is a modified DNH-SaW which improves 
wait phase of DNH-SaW protocol base on delivery predictability using PROPHET. This scheme improves average latency and gives better delivery ratio as compared to BSW and DNH-SaW. This scheme uses node performance of receiver node to forward number of message copies in spray phase and delivery predictability based forwarding in wait phase to improve average latency.

Results shows that the proposed scheme reduces average latency of DNH-SaW and also BSW while achieving a better delivery ratio as compared to both BSW and DNH-SaW. The overhead ratio of the proposed scheme is slightly higher than the other two protocols but delivery ratio which is an important metric in DTNs has improved significantly. In future this work will be extended to improve Modified DNHSaW using PROPHET to reduce average latency.

\section{REFERENCES}

[1] Fall, K. "A Delay-Tolerant Network architecture for challenged Inter-nets", In ACM SIGCOMM, 2003.

[2] Cerf, V., Burleigh, S., and Dust, R. 1995. "Interplanetary internet (IPN): Architectural Definition" Phil. Trans. Roy. Soc. London, vol. A247, 529-551.

[3] Dunbabin, M., Corke, P., Vailescu, I., and Rus, D. 2006. Data muling over underwater wireless sensor networks using an autonomous underwater vehicle. In Proceedings of the Intl Conf on Robotics and Automation (ICRA) IEEE.

[4] Juang, P., Oki, H., and Wang, Y. 2002. Energy-efficient computing for wildlife tracking: design tradeoffs and early experiences with zebranet. In Proceedings of the ASPLOS '02, Oct. 2002.

[5] Ivancic, W., Eddy, W., and Wood, L. 2008. Delay/Disruption-tolerant network testing using a LEO satellite". In Proceedings of the ESTC, Maryland.

[6] Warthman, F., 2008. Delay Tolerant Networks (DTNs), a tutorial.

[7] Lindgren, A., Doria, A., and Schein, O., Probabilistic routing in intermittently connected networks. Lecture Notes in Computer Science (including subseries Lecture Notes in Artificial Intelligence and Lecture Notes in Bioinformatics), pp. 239-254.
[8] Spyropoulos, T., Psounis, K., and Raghavendra, C. S. "Spray and Wait: An efficient routing scheme for intermittently connected mobile networks", in ACM, 2005.

[9] Prueksasri, V., Intanagonwiwat, C., and Rojviboonchai, K. 2011. DNH-SaW: The Different neighbor-history spray and wait routing scheme for delay tolerant networks. In Proceedings of the International Symposium on Intelligent Signal Processing and Communication Systems (ISPACS).

[10] Jenjaturong, S., and Intanagonwiwat, C. 2008. A set cover-based density control algorithm for sensing coverage problems in wireless sensor networks. In Proceedings of the 3rd International Conference on Cognitive Radio Oriented Wireless Networks and Communications, CrownCom.

[11] Xue, J., Fan, X., Cao, Y., Fang J. and Li, J. 2009. Spray and wait routing based on average delivery probability in delay tolerant network. In Proceedings of the International Conference on Networks Security, Wireless Communications and Trusted Computing, Wuhan, Hubei, 500 - 502.

[12] Kim, Y. P., Koo, J., Jung, E., Nakano, K., Sengoku, M., and Park, Y. J. 2010. Composite methods for improving spray and wait routing protocol in delay tolerant Networks. In Proceedings of the International Symposium on Communications and Information Technologies (ISCIT), Tokyo, 1229 - 1234.

[13] Wang, G., Wang, B., and Gao, Y. 2010. Dynamic spray and wait routing algorithm with quality of node in delay tolerant network. In Proceedings of the International Conference on Communications and Mobile Computing.

[14] Keranen, A., Ott, L., and Karkkainen, T. 2009. The ONE simulator for DTN protocol evaluation. In Proceedings of the 2nd International Conference on Simulation Tools and Techniques, Belgium.

[15] Mehto A., and Chawla, M. 2013. Comparing delay tolerant network routing protocols for optimizing Lcopies in spray and wait routing for minimum delay. In Proceedings of the World Conference on Advances in Communication and Control Systems,(CAC2S), India. 